\title{
In Memoriam: Gisbert Winnewisser
}

\author{
Eric Herbst ${ }^{1}$, Pierre Encrenaz ${ }^{2}$, and Rafael Bachiller ${ }^{3}$ \\ ${ }^{1}$ University of Virginia and Ohio State University, USA \\ ${ }^{2}$ Observatoire de Paris, France \\ ${ }^{3}$ Observatorio Astronómico Nacional, Spain
}

Gisbert Winnewisser, emeritus Professor of Physics at the University of Köln, passed away in March 2011 after a long illness. His dedication to molecular spectroscopy in the laboratory and in the interstellar medium, coupled with his very influential voice for molecular science will be extremely difficult to replace.

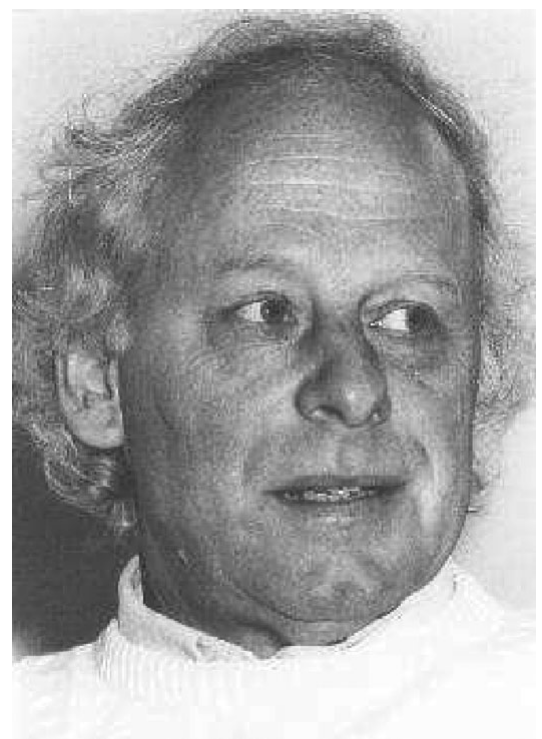

Gisbert Winnewisser

Born in Karlsruhe on September 7, 1936, Gisbert moved with his family to a farm in the Black Forest where they lived until 1946. Gisberts brother Manfred, his twin sister Ingrid, and Gisbert lived simply and independently with their mother, after their father died near Moscow. This tragic event marked Gisbert deeply. The family went back to Karlsruhe in 1946 where Gisbert graduated from gymnasium in 1956. He then became a physics student at the Technical University of Karlsruhe and graduated with a Master of Science degree in 1963. His brother Manfred had then become a post-doctoral fellow in the laboratory of Walter Gordy, a well-known molecular spectroscopist whose book on the subject, co-authored with Cook, is still known as a bible in the subject. Gisbert decided to follow the same career path, and became a $\mathrm{PhD}$ student under Professor Gordy at Duke University in Durham, North Carolina where he stayed until 1968. In the interim, he married Helga ter Jung in 1964 in Karlsruhe, and the couple stayed in Durham where their son Carsten was born in 1966. Gisbert defended his PhD thesis in 1967, the topic of which is the rotational-torsional spectrum of the internal rotor molecule $\mathrm{HSSH}$, an intriguing molecule on which he kept working for decades, along with similar species such as $\mathrm{HOOH}$ and $\mathrm{HSOH}$. 
Gisbert and his family then moved to Ottawa, where Gisbert had been appointed a postdoctoral associate in the world- famous spectroscopic laboratory of Gerhard Herzberg. He worked there until 1972 with a short stay in Vancouver at the University of British Columbia. This was the time of the first observations of interstellar molecules: $\mathrm{H}_{2} \mathrm{O}, \mathrm{NH}_{3}$, $\mathrm{H}_{2} \mathrm{CO}, \mathrm{CO}, \mathrm{CN}$, OCS, etc. Gisbert realized what could be done in this new field with his knowledge of spectroscopy, and he accepted a permanent position at the Max Planck Institute for Radioastronomy in Bonn as a molecular radio astronomer. He spent thousands of hours observing the interstellar medium and discovered formic acid, HNC, and vinyl cyanide, as well as isotopomers of HCCCN. He also participated in the earliest studies of TMC1, now the best-known cold interstellar cloud core. Gisbert also collaborated with his brother Manfred, then professor of physics at Justus Liebig University in Giessen, and his sister-in-law Brenda in assorted spectroscopic projects. He started to defend the idea of a large submillimeter antenna in space as a member of ESA's Astrophysics Working Group.

In 1979, he was offered and accepted a chair in Physics at the University of Köln, and he became Director of the I. Physikalishes Institute. Gisbert made of this Institute one of the worlds leading centers for high-resolution molecular spectroscopy, developing new instrumentation for ground and space applications, including acousto-optical detectors, local oscillators, SIS junctions, and new submillimeter spectrometers. His laboratory provided unique equipment for the satellites SWAS, Herschel, and for ASTRO (the Antarctic Submillimeter Telescope and Remote Observatory). The transfer of carcinotron techniques from Russia to his laboratory, where it was used with his OROTRON spectrometer, kept alive this technology.

Gisbert built a submillimeter telescope in Köln and then moved it to the Gornergrat, near Zermatt in southern Switzerland. The telescope, known as KOSMA, helped to educate many students in high-frequency radio astronomy. Indeed, the number of spectroscopy and astronomy students who received and continue to receive their degrees at the I. Physikalisches Institute is exceedingly impressive.

Gisbert was a major figure on the international scene and helped to promote interstellar molecular astronomy. The series of Zermatt conferences on the latest discoveries in the interstellar medium and in the laboratory attracted many well-known and younger spectroscopists and molecular astronomers. He also co-founded the LEA Hires program (Laboratoire Européen Associé for High Resolution Spectroscopy), a European association of spectroscopy laboratories in Germany, France, Belgium and Spain, specializing in complementary techniques and theories.

Gisbert is the author or coauthor of more than 400 publications, and has received many international awards, including the Honorary Medal of the University of Helsinki (1985), the Max Planck Research Prize (1993), the Philip Morris Prize (1994), the Honorary Medal of the Ioannes Marcus Marci Spectroscopic Society (1996), and the Historical Medal of the Charles University in Prague (2000). NASA awarded him a Group Achievement Award for his contribution to the SWAS satellite.

On behalf of all Gisbert Winnewisser's students, colleagues, associates, coauthors, and friends, we dedicate this volume to his memory. 